\title{
Dynamics of Confined Microgel Liquids: Weakened Spatial Confinement Effect by Microgel Particle Compliance
}

\author{
Raymond P. Seekell, III, ${ }^{\mathrm{a}}$ Kehua Lin, ${ }^{\mathrm{b}}$ and Yingxi Zhu ${ }^{\mathrm{b}, *}$ \\ ${ }^{a}$ LiquiGlide, Inc., 75 Sidney Street, Cambridge, MA 02139, USA \\ ${ }^{b}$ Department of Chemical Engineering and Materials Science, 5050 Anthony Wayne Drive, \\ Wayne State University, Detroit, MI 48202, USA
}

\section{Supporting Information}

Figure 1. (a) Schematic diagram of home-built compression apparatus that is mounted on the sample stage of an inverted confocal microscope. (b)Representative fluorescence micrograph of the midplane of PNIPAM microgels confined between two solid surfaces. Scale bar is $5 \mu \mathrm{m}$.

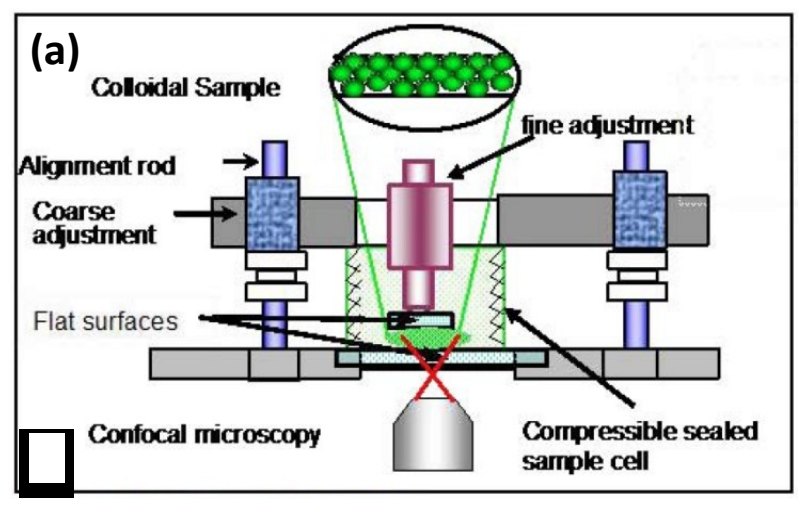

(b)

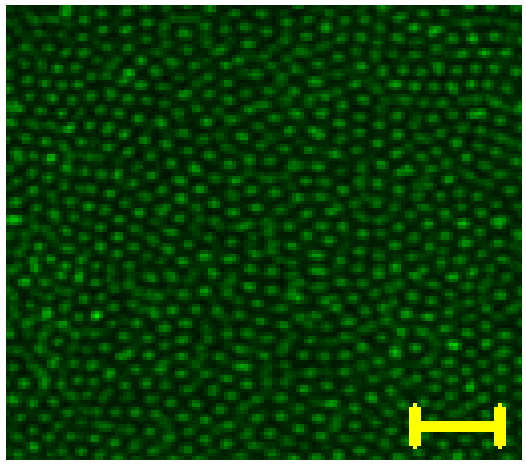


Figure 2. Mean squared displacement (MSD) against lag time, $\tau$ for confined PNIPAM microgel liquids of $E_{p}=22 \mathrm{kPa}$ at $\phi=0.56$ at varied gap spacing, $H / d_{H}=85$ (orange squares), 43 (blue squares), 21 (red squares), and 14 (green squares). At the low to intermediate lag time range, extended plateau in MSD indicates dynamic rest of confined microgel particles. Combined with reduced particle mobility, the results suggest confinement-induced glass dynamics of confined stiff microgels, which is consistent with the observed confinement-induced glass transition of model "hard-sphere" dense colloidal liquids.

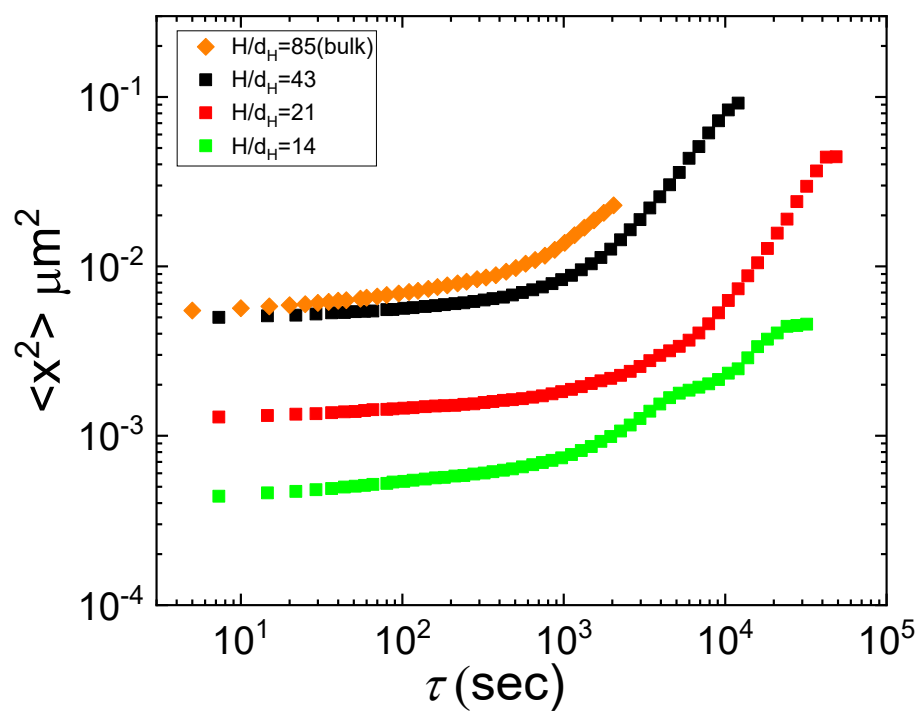


Figure 3. Pair correlation function, $g(r)$ of confined PNIPAM microgel liquids of $E_{p}=22 \mathrm{kPa}$ and $\phi=0.49$ between two solid surfaces of varied gap spacing, $H / d_{H}=43$ (black line), 20 (red line), and 9 (green line).

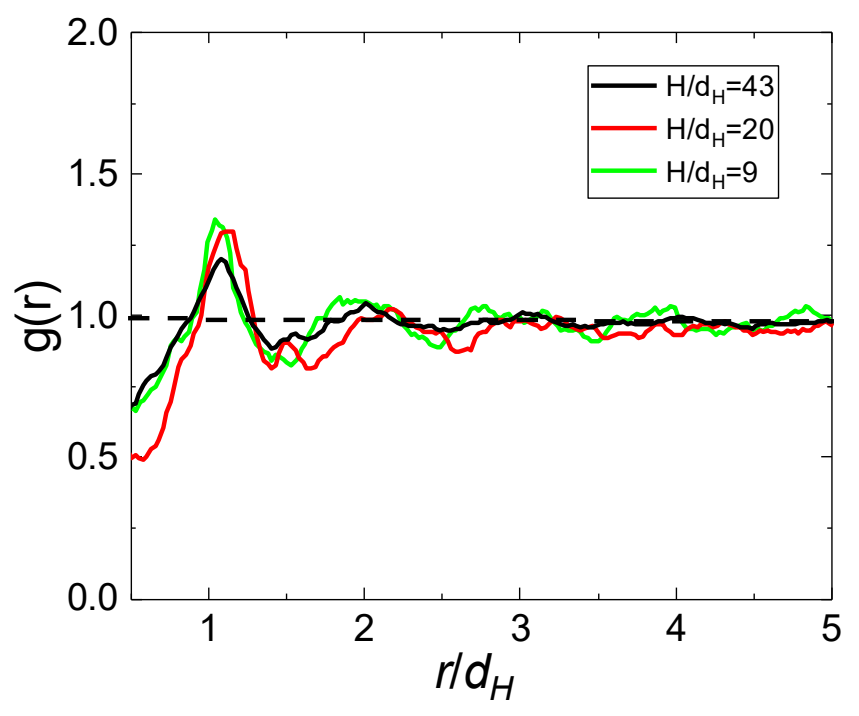

Figure 4. Overlap order parameter, $\mathrm{q}_{\mathrm{s}}(\tau)$ of confined PNIPAM microgel liquid of $E_{p}=169 \mathrm{~Pa}$ and $\phi=0.49$ at varied gap spacing, $\mathrm{H} / \mathrm{d}_{\mathrm{H}}=85$ (black squares), 16 (red circles), and 11 (green triangles).

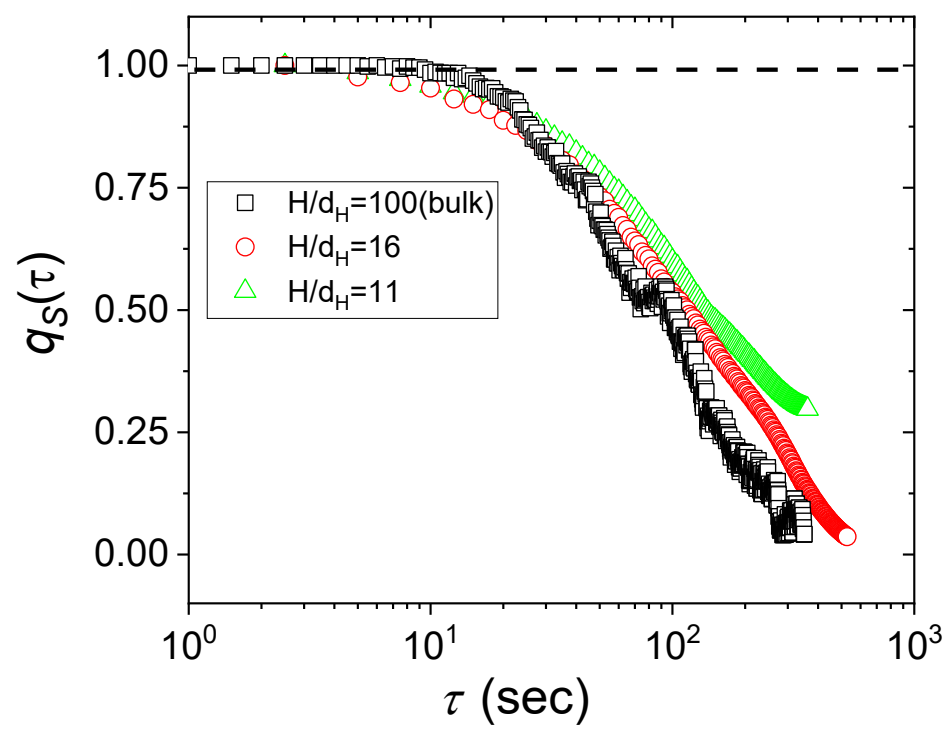


Figure 5. Overlap order parameter, $\mathrm{q}_{\mathrm{s}}(\tau)$ of confined PNIPAM microgel liquid of $E_{p}=22 \mathrm{kPa}$ and $\phi=0.49$ at varied gap spacing, $\mathrm{H} / \mathrm{d}_{\mathrm{H}}=43$ (black squares), 20 (red circles), and 9 (green triangles).

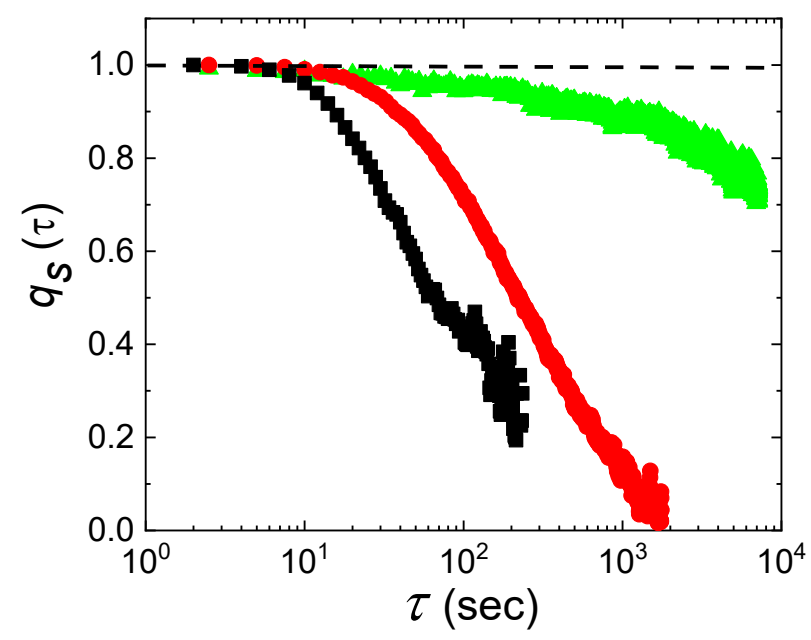

Figure 6. Normalized $\alpha$-relaxation time, $\tau_{\alpha}$ by $\tau_{\alpha, \text { bulk }}$ of confined PNIPAM microgel liquid of $E_{p}=22 \mathrm{kPa}$ and $\phi=0.49$ against $H / d_{H}$. The interception of the extrapolation of $\tau_{\alpha} / \tau_{\alpha, \text { bulk }}$ at small $\mathrm{H} / \mathrm{d}_{\mathrm{H}}$ with the unity determines $\left(H / d_{H}\right)_{c r}$. Inset: $\mathrm{H} / \mathrm{d}_{\mathrm{H}}$-dependent $\tau_{\alpha}$.

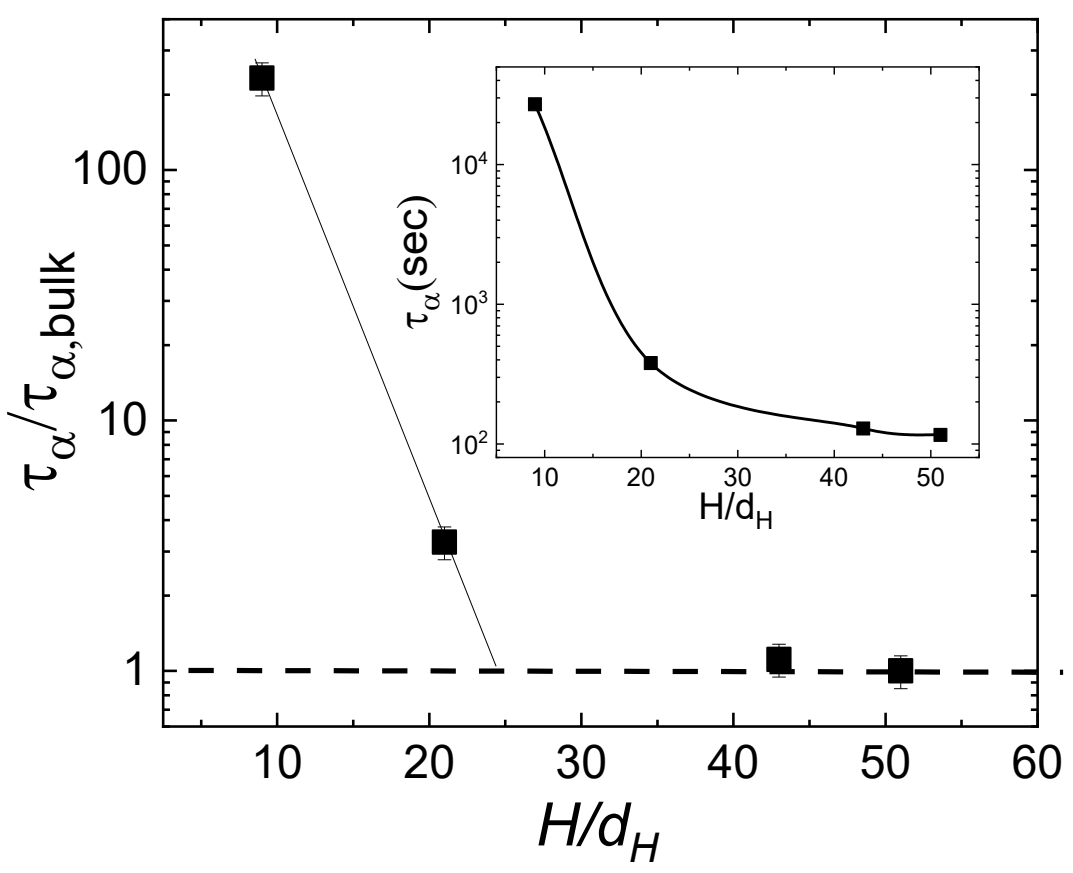

